\section{GENETIC POLYMORPHISM +1444CT IN THE C - REACTIVE PROTEIN IS ASSOCIATED WITH THE SUSCEPTIBILITY FOR SYSTEMIC LUPUS ERYTHEMATOSUS AND DISEASE ACTIVITY}

${ }^{1} \mathrm{EM}$ Vissoci Reiche*, ${ }^{2} \mathrm{~F}$ Delongui, ${ }^{1} \mathrm{MAB}$ Lozovoy, ${ }^{3} \mathrm{TMV}$ Iriyoda, ${ }^{2} \mathrm{DF}$ Alfieri, ${ }^{2} \mathrm{~T}$ Flauzino, ${ }^{4}$ I Dichi, ${ }^{1}$ ANC Simão. ${ }^{1}$ Health Sciences Center- State University of Londrina- LondrinaParaná- Brazil-, Pathology- Clinical Analysis and Toxicology, Londrina, Brazil; ${ }^{2}$ Health Sciences Postgraduate Program- Health Sciences Center- State University of LondrinaLondrina- Paraná- Brazil-, Pathology- Clinical Analysis and Toxicology, Londrina, Brazil; ${ }^{3}$ Outpatient Clinic for Rheumatology- University Hospital- State University of LondrinaLondrina- Paraná-Brazil, Clinical Medicine, Londrina, Brazil; ${ }^{4}$ Health Sciences Center-State University of Londrina- Londrina- Paraná-Brazil-, Clinical Medicine, Londrina, Brazil

10.1136/lupus-2017-000215.327

Background and aims The gene C-reactive protein (CRP), located at 1q23-24, is a candidate to be investigated as a susceptibility locus for systemic lupus erythematosus (SLE). The aim of the study was to evaluate the association between the +1444CT CRP polymorphism with the susceptibility for SLE, disease activity, and CRP serum levels.

Methods The study enrolled 176 SLE patients and 223 healthy controls from Brazilian population. SLE disease activity (SLEDAI), clinical and laboratorial characteristics were evaluated. The +1444CT CRP polymorphism was determined using polymerase chain reaction and restriction fragment length polymorphism.

Results The frequency of CC vs. TT genotypes and the C vs. $\mathrm{T}$ allele among the patients differed from the controls $(p=0.0201$ and $p=0.0072$, respectively). Patients carrying the $T$ allele presented higher CRP $(p=0.017)$ and showed a trend toward higher IL-6 compared with patients carrying the C allele $(p=0.057)$. The increased CRP was independently of the IL-6 in these subgroups of patients. SLE patients carrying the CC genotype showed positive correlation between CRP and C4 levels $(p=0.039)$, while those with $T$ allele presented a trend toward a negative correlation between CRP and C3 and C4 $(p=0.056$ and $p=0.073$, respectively); and a trend toward positive correlation with anti-nucleosome and anti-dsDNA $(\mathrm{p}=0.052$ and $\mathrm{p}=0.091$, respectively).

Conclusions Our data showed that +1444CT CRP polymorphism was associated with SLE susceptibility and CRP levels, as well as CRP levels were associated with disease activity, suggesting that this polymorphism may play a role in the pathophysiology of SLE, which may be used as a possible marker of disease activity.

\section{CCR5 $\delta 32$ (RS333) POLYMORPHISM IS ASSOCIATED WITH SUSCEPTIBILITY TO SYSTEMIC LUPUS ERYTHEMATOSUS IN FEMALE BRAZILIAN PATIENTS}

${ }^{1}$ EM Vissoci Reiche*, ${ }^{2}$ THL Baltus, ${ }^{2}$ AP Kallaur, ${ }^{1}$ MAB Lozovoy, ${ }^{1}$ HK Morimoto, ${ }^{2} \mathrm{~F}$ Delongui, ${ }^{2}$ DF Alfieri, ${ }^{3}$ TMV Iriyoda, ${ }^{4}$ I Dichi, ${ }^{1}$ ANC Simão. ${ }^{1}$ Health Sciences Center- State University of Londrina- Londrina- Paraná- Brazil-, Pathology- Clinical Analysis and Toxicology, Londrina, Brazil; ${ }^{2}$ Health Sciences Postgraduate Program-Health Sciences Center- State University of Londrina- Londrina- Paraná- Brazil-, Pathology- Clinical Analysis and Toxicology, Londrina, Brazil; ${ }^{3}$ Outpatient Clinic for Rheumatology- University Hospital- State University of Londrina- Londrina- Paraná- Brazil, Clinical Medicine, Londrina, Brazil; ${ }^{4}$ Health Sciences Center- State University of Londrina- Londrina- Paraná- Brazil-, Clinical Medicine, Londrina, Brazil

\subsection{6/lupus-2017-000215.328}

Background and aims The role of CCR5 32 (rs333) polymorphism in the pathogenesis of systemic lupus erythematosus
(SLE) has been evaluated worldwide. The aim of this study was to determine the association between CCR $5 \Delta 32$ polymorphism with the susceptibility to SLE and the activity of disease in female Southern Brazilian patients.

Methods The study enrolled 169 female SLE patients and 132 unrelated female healthy individuals. Baseline clinical, laboratorial characteristics, and the SLE activity (determined using the SLEDAI) were evaluated according to the CCR5 $\Delta 32$ genotypes. The CCR5 32 polymorphism was determined from genomic DNA using a polymerase chain reaction.

Results The frequencies of the genotypes CCR5/CCR5, CCR5/ CCR5 532 and CCR5 $\triangle 32 / C C R 5 \Delta 32$ were $87.6 \%, 11.8 \%$, and $0.6 \%$, respectively, among the patients, and $96.2 \%, 3.8 \%$, and $0.0 \%$, respectively among the controls, $[p=0.0116$, odds ratio:3.432 (95\% confidence interval:1.252-9.40). Patients carrying the CCR5/CCR5 $\Delta 32$ and CCR5 $32 / C C R 5 \Delta 32$ genotypes presented earlier age of onset of disease $(p=0.0293)$ and higher levels of anti-dsDNA $(p=0.0255)$, than those carrying the wild type genotype. When the analysis was adjusted for ethnicity, only the age at onset of disease remained associated with the CCR5 32 polymorphism $(\mathrm{p}<0.05)$; patients with variant CCR5 432 allele (heterozygous and homozygous), presented lower age at onset of disease than those with the wild type genotype.

Conclusions The results suggest that the CCR5 432 polymorphism might be associated with SLE genetic predisposition among female Brazilian patients and the age at onset of the disease; however, this genetic variant was not associated with the activity of SLE in this population.

\section{ELEVATED IFIT3 IN MONOCYTES CONTRIBUTED TO HYPERACTIVE CGAS-STING SIGNALLING PATHWAY IN SLE}

J Wang*, B Qu, N Shen. Ren Ji Hospital- School of Medicine- Shanghai Jiao Tong University, Rheumatology, Shanghai, China

\subsection{6/lupus-2017-000215.329}

Background and aims IFIT3 is one of the Interferon-stimulated genes showed significantly increase in PBMCs of SLE patients. However, the functions of IFIT3 in dysregulated immune responses of SLE are not fully understood. SLE is featured by over production of nuclear antigens, such as dsDNAs, from debris of numerous dead cells, which give rise to autoantibody production. cGAS-STING signalling pathway has been proposed to play an important role in sensing DNA and producing inflammatory cytokines in SLE. Our study is IFIT3's function in regulating cGAS-STING signalling pathway in SLE. Methods Monocytes were isolated from SLE patients or healthy controls by Ficoll-paque method and CD14+ magnetic beads. The expression of IFN $\beta$ and phosphorylation of IRF3 were measured in either IFIT3 over-expressing or knockout cells upon VACV-70 stimulation by Q-PCR and Western blot. We used Co-IP to identify the interaction between IFIT3 and its interaction proteins.

Results cGAS-STING signalling pathway was over-activated in monocytes from SLE patients compared to healthy controls. The expression of IFIT3 was significantly elevated in SLE patients and was positively correlated with the activity of cGAS-STING signalling pathway. In vitro, we revealed that the expression of IFN $\beta$ and phosphorylation of IRF3 could be reduced by knocking down IFIT3, while over-expression of 
IFIT3 produced an opposite effect. Mechanistically, IFIT3 protein was found to interact both with STING and TBK1.

Conclusions We proposed that cGAS-STING signalling pathway was hyperactive in monocytes of SLE. IFIT3 is one of the important genes which contributed to the over-activation of cGAS-STING signalling pathway and over-produced IFN in SLE pathogenesis.

\section{MASS SPECTROMETRIC SEQUENCING OF PRECIPITATING ANTI-RO REVEALS UNIQUE VH/VL PEPTIDE BIOMARKERS}

${ }^{1} \mathrm{JJ}$ Wang*, ${ }^{1} \mathrm{M}$ Al kindi, ${ }^{1} \mathrm{~A}$ Colella, ${ }^{1} \mathrm{~L}$ Dykes, ${ }^{1} \mathrm{M}$ Jackson, ${ }^{2} \mathrm{~T}$ Chataway, ${ }^{3} \mathrm{~J}$ Reed, ${ }^{1} \mathrm{~T}$ Gordon. ${ }^{1}$ Flinders University, Immunology, Adelaide, Australia; ${ }^{2}$ Flinders University, Flinders Proteomic Facility, Adelaide, Australia; ${ }^{3}$ Garvan Institute of Medical Research, Immunology Division- Immunogenomics Laboratory, Sydney, Australia

\subsection{6/lupus-2017-000215.330}

Background and aims Autoantibodies directed against the 60$\mathrm{kD}$ Ro (Ro60)/SSA ribonucleoprotein particle are the major target of humoral autoimmunity in patients with systemic lupus erythematosus (SLE) and primary Sjögren's syndrome (SS). However, little is known of the anti-Ro60 immunoglobulin variable-region $(\mathrm{IgV})$ repertoire in terms of clonality and $\mathrm{IgV}$ gene usage at the level of the serum proteome.

Methods We used high-resolution mass spectrometry to sequence precipitating anti-Ro60 proteomes from sera of patients with SLE and primary SS and compare $\operatorname{IgV}$ peptide signatures in Ro/La autoantibody subsets. Anti-Ro60 were purified by elution from native Ro60-coated ELISA plates and subjected to combined de novo amino acid sequencing and database matching. Additionally, Ro60 precipitins from counterimmunoelectrophoresis gels were excised, digested and sequenced directly by mass spectrometry.

Results Anti-Ro60 Igs purified from ELISA plates and Ro60 precipitins were comprised dominant public sets of IgG1 kappa and lambda restricted heavy and light chains (with sharing of IGHV3-23, IGHV3-74 and IGHV1-18; IGKV3-20, IGKV1-5 and IGLV3-19). Significantly, mass spectrometric sequencing of purified anti-Ro60 IgGs from SLE patients showed the same convergence of autoantibody repertoires as primary SS, apart from one SLE patient who lacked IGHV374 , suggesting that humoral anti-Ro60 molecular signatures are conserved across these two systemic autoimmune diseases. Specific IgV amino acid substitutions stratified anti-Ro60 from anti-Ro60 plus anti-La responses, providing a molecular fingerprint of Ro60/La determinant spreading.

Conclusions Unique anti-Ro60 $\mathrm{IgV}$ peptide signatures provide insights in to mechanisms of autoantibody production as well as holding promise as serum-based molecular markers for clinical syndromes linked to Ro60 autoimmunity.

\section{IL-1B AND IL-6 ARE HIGHLY EXPRESSED IN RF+IGE+ SYSTEMIC LUPUS ERYTHEMATOUS SUBTYPE}

${ }^{1} Y W_{u^{*}},{ }^{1} \mathrm{~B}$ Cai, ${ }^{1} \mathrm{~J}$ Zhang, ${ }^{1} \mathrm{~B}$ Shen, ${ }^{1} \mathrm{Z}$ Huang, ${ }^{2} \mathrm{C}$ Tan, ${ }^{3} \mathrm{CC}$ Baan, ${ }^{1} \mathrm{~L}$ Wang ${ }^{*} .{ }^{1}$ West China Hospital affiliated Sichuan University, Department of Laboratory Medicine, Chengdu, China; ${ }^{2}$ West China Hospital affiliated Sichuan University, Department of Rheumatology, Chengdu, China; ${ }^{3}$ West China Hospital affiliated Sichuan University, Department of Laboratory Medicine, Rotterdam, Netherlands Antilles
Background and aims Systemic lupus erythematous (SLE) is anautoimmune disease with great heterogeneity in pathogenesis and clinical symptoms. To better categorise SLE subtypes we determined the dominant cytokines based on $\mathrm{RF}+\mathrm{IgE}+$ (both $\mathrm{RF}$ and IgE were positive ) familial SLE.

Methods RF, IgE and multiple cytokines (i.e., IL-1 $\beta$, IL-6, IL8 , IL-10, IL-17, IFN- $\gamma$, IP-10, MCP-1 andMIP-1 $\beta$ ) were measured in sera of familial SLE $(n=3)$, non-inherited SLE $(n=108)$ and healthy controls $(\mathrm{n}=80)$.

Results Three SLE patients in family and 5 out of 108 noninherited patients featured with $\mathrm{RF}+\mathrm{IgE}+$. These $\mathrm{RF}+\mathrm{IgE}$ + SLE patients expressed significantly higher levels of IL-1 $\beta$ and IL-6 than the other SLE patients $(\mathrm{p}<0.05)$. IL-6 correlated with both $\operatorname{IgE}$ and IL-1 $\beta$ levels in $\mathrm{RF}+\mathrm{IgE}+\mathrm{SLE}$ patients $\left(\mathrm{r}^{2}=0.583, \mathrm{p}=0.027 ; \mathrm{r}^{2}=0.847, \mathrm{p}=0.001\right)$.

Conclusions Both IL-1 $\beta$ and IL- 6 are highly expressed cytokines in $\mathrm{RF}+\mathrm{IgE}+\mathrm{SLE}$ subtype which may be related to the pathogenesis of this special SLE subtype.

\section{CEREBROSPINAL FLUID UBIQUITIN CARBOXYL HYDROLASE L1 (UCH-L1) AND ITS AUTOANTIBODY ARE USEFUL BIOMARKERS FOR NEUROPSYCHIATRIC SYSTEMIC LUPUS ERYTHEMATOSUS (NPSLE)}

${ }^{1} \mathrm{X} \mathrm{Li}^{*},{ }^{2} \mathrm{Y}$ Meng, ${ }^{1} \mathrm{Y}$ Gan, ${ }^{1} \mathrm{G}$ Wang, ${ }^{1} \mathrm{R} \mathrm{Mu},{ }^{1} \mathrm{X}$ Liu, ${ }^{1} \mathrm{X}$ Sun, ${ }^{1} \mathrm{Z} \mathrm{Li} .{ }^{1}$ peking university people's hospital, rheumatology and immunology, beijing, China; ${ }^{2}$ The Fifth Affiliated Hospital of Zhengzhou University, Department of Rheumatology and Immunology, Zhengzhou, China

\subsection{6/lupus-2017-000215.332}

Background and aims To identify cerebrospinal fluid (CSF) biomarkers for the diagnosis and disease severity evaluation of neuropsychiatric systemic lupus erythematosus (NPSLE).

Methods CSF samples (36 NPSLE, 19 SLE controls, 4 other connective tissue disease controls and 10 other nervous system disease controls) and serum samples (21 NPSLE and 6 SLE controls) were included in this study. The levels of UCH-L1 and its autoantibody were determined by Luminex multiplex (xMAP) assays and enzyme-linked immunosorbent assay (ELISA) respectively.

Results 1) Among 6 candidate neurological disease related proteins, including ubiquitin carboxyl hydrolase L1 (UCH-L1), total Tau protein, phospho-Tau protein, DJ-1 protein, nerve growth factor (NGF) and $\alpha$-Synuclein $(\alpha-S Y N)$, UCH-L1 was significantly elevated in the CSF of patients with NPSLE defined by 2001 Ainiala's modified criteria, while it was lower in those defined by 1999 ACR criteria but merely presented with headache or mild mood disorder, and in SLE controls whose neuropsychiatric manifestations were not due to SLE. The elevation of CSF UCH-L1 levels were associated with elevated SLEDAI and the number of NPSLE manifestations diagnosed in individual patients. 2) The CSF levels of UCH-L1 autoantibodies were significantly elevated in patients with NPSLE, and showed a sensitivity of $53 \%$ and a specificity of $91 \%$ for the diagnosis of NPSLE. CSF anti-UCH-L1 levels were associated with organ involvements, and were positively correlated with serum anti-UCH-L1 levels in the NPSLE patients.

Conclusions Anti-UCH-L1 is a promising CSF biomarker for NPSLE diagnosis with high sensitivity and specificity, and CSF levels of UCH-L1 may reflect the severity of NPSLE. 